\title{
Injection Site Reaction, CTCAE
}

National Cancer Institute

\section{Source}

National Cancer Institute. Injection Site Reaction, CT CAE. NCI Thesaurus. Code C143585.

A disorder characterized by an intense adverse reaction (usually immunologic) developing at the site of an injection. 